\title{
COMPARAÇÃO CLÍNICA E ANATÔMICA DO NERVO FIBULAR NA ZONA DE SEGURANÇA DE GERDY
}

\author{
CLINICAL AND ANATOMICAL COMPARISON OF THE FIBULAR NERVE IN THE GERDY SAFE ZONE
}

Pedro José Labronici', Thiago Martins Teixeira², Fernando Barone de Medeiros², José Sergio Franco ${ }^{3}$,

Rolix Hoffmann ${ }^{4}$, Marco Aurélio Fonseca Passos ${ }^{5}$, Paulo Roberto Barbosa de Toledo Lourenço ${ }^{6}$,

Hélio Jorge Alvachian Fernandes ${ }^{7}$, Fernando Baldy dos Reis ${ }^{8}$

\section{RESUMO}

Objetivo: Comparar clínica e anatomicamente os limites do nervo fibular na zona de segurança de Gerdy em cadáveres. Métodos: Foram utilizados 50 espécimes anatômicos de joelhos e medidos clinicamente (antes de dissecção) para determinar as distâncias e ângulos entre: o tubérculo de Gerdy e a região posterior da fíbula $(\mathrm{cm})$, ângulo entre a linha correspondente à região posterior da fíbula e a crista tibial (graus) e o ângulo entre o tubérculo de Gerdy e crista tibial (graus). Após a dissecção das peças anatômicas, os joelhos foram novamente medidos, para determinar as distâncias e ângulos entre: o tubérculo de Gerdy e o nervo fibular (cm), ângulo entre o nervo fibular, na região posterior da fíbula e a crista tibial (graus) e o ângulo entre o tubérculo de Gerdy e crista tibial (graus). Resultados: Existe aumento significativo da distância entre o tubérculo de Gerdy e a região posterior da fíbula $(\mathrm{cm})$, após dissecção, em média $0,26 \mathrm{~cm}$ ( $p=0,018)$, o que corresponde a 8,6\% $(p=0,007)$ e também existe diminuição significativa do ângulo entre a região posterior da fíbula e a crista tibial após dissecção, em média de $3^{\circ}(p=0,047)$, que corresponde a 2,1\% $(p=0,06)$. Conclusão: Apesar da diferença antes e depois da dissecção, a zona de segurança de Gerdy pode ser considerada segura para os procedimentos ortopédicos, na região proximal da tíbia, evitando danos ao nervo fibular e seus ramos.

Descritores - Nervo fibular; Dissecção; Cadáver; Tíbia

\section{ABSTRACT}

Objective: to clinically and anatomically compare the limits of the fibular nerve in Gerdy's Safe Zone in cadavers. Methods: Fifty anatomical knee specimens were clinically measured (before dissection) to determine the distances and angles between: Gerdy's tubercle and the posterior region of fibula $(\mathrm{cm})$; the angle between the line corresponding to the posterior region of fibula and the tibia crest (degrees); and the angle between Gerdy's tubercle and tibial crest (degrees). After dissection of the anatomical parts, the knees were measured again, to determine the distances and angles between: Gerdy's tubercle and the fibular nerve $(\mathrm{cm})$, the angle between the fibular nerve, in the posterior region of the fibula, and the tibial crest (degrees), and the angle between Gerdy's tubercle and the tibial crest (degrees). Results: There was a significant increase in the distance between Gerdy's tubercle and the posterior region of fibula $(\mathrm{cm})$, after dissection, of an average $0.26 \mathrm{~cm}(p$ $=0.018)$, corresponding to $8.6 \%(p=0.007)$. There was also a significant reduction in the angle between the posterior region of fibula and the tibial crest after dissection, of an average $3^{\circ}(p=$ 0.047), corresponding to 2.1\% ( $p=0.06)$. Conclusion: Despite the difference before and after dissection, Gerdy's Safety Zone can be considered safe for orthopedic procedures involving the proximal region of the tibia, preventing damage to the fibular nerve and its branches.

Keywords - Fibular nerve; Dissection; Cadaver; Tibia

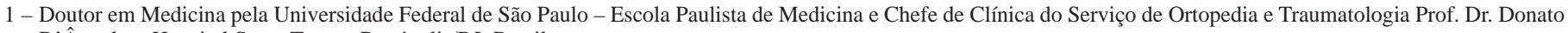
D’Ângelo - Hospital Santa Teresa, Petrópolis/RJ, Brasil.

2 - Médico Residente em Ortopedia e Traumatologia do Serviço de Ortopedia e Traumatologia Prof. Dr. Donato D’Ângelo - Hospital Santa Teresa, Petrópolis/RJ, Brasil.

3 - Professor Associado e Chefe do Departamento de Ortopedia e Traumatologia da Faculdade de Medicina da UFRJ/RJ, Brasil.

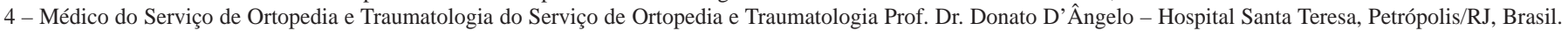

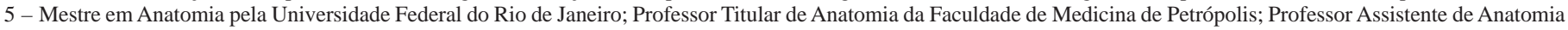
e Médico da Universidade do Estado do Rio de Janeiro/ Brasil.

6 - Médico Responsável pelo Grupo de Trauma do Hospital de Ipanema, Rio de Janeiro, Brasil.

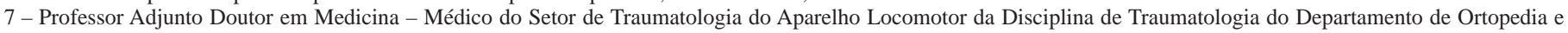
Traumatologia da Unifesp/EPM, São Paulo, Brasil.

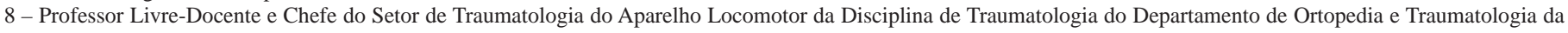
Unifesp/EPM, São Paulo, Brasil.

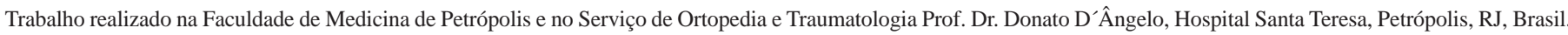
Correspondência: Av. Roberto Silveira, 187, apto. 601 - 25685-040 - Petrópolis, RJ. E-mail: plabronici@globo.com 


\section{INTRODUÇÃO}

A anatomia cirúrgica do nervo fibular e sua distribuição têm sido bem documentadas ${ }^{(1-5)}$. Entretanto, quando comparadas na dissecção anatômica, foi observado serem descrições puramente genéricas do trajeto do nervo ${ }^{(4)}$. A maioria desses estudos tem descrito o nervo e seus ramos em relação à cabeça da fíbula de forma linear e bidimensional, em relação à região proximal da tíbia, fazendo com que a relação espacial do nervo seja difícil de entender ${ }^{(4,5)}$. O nervo fibular fornece inervação motora para os músculos do compartimento anterior da perna. As duas mais importantes funções são a dorsoflexão do tornozelo e extensão dos dedos, que são ambos os componentes principais da marcha normal. Na região proximal da tíbia, lesões do nervo fibular podem produzir graves complicações $^{(6-8)}$.

O objetivo deste estudo foi comparar clínica e anatomicamente os limites do nervo fibular na zona de segurança de Gerdy.

\section{MÉTODOS}

Foram utilizados 50 joelhos de cadáver da Faculdade de Medicina de Petrópolis/RJ. Foram excluídos os joelhos com deformidades ou dissecção prévias e sinais de fraturas. Dos espécimes anatômicos, 24 eram do lado direito e 26 do esquerdo e 45 eram do sexo masculino e cinco do feminino. Os espécimes anatômicos dos joelhos foram medidos clinicamente (antes de dissecção) para determinar as distâncias e ângulos entre: o tubérculo de Gerdy e a região posterior da fíbula $(\mathrm{cm})$, ângulo entre a linha correspondente à região posterior da fíbula e a crista tibial (graus) e o ângulo entre o tubérculo de Gerdy e crista tibial (graus). Após a dissecção das peças anatômicas, os joelhos foram novamente medidos, para determinar as distâncias e ângulos entre: o tubérculo de Gerdy e o nervo fibular (cm), ângulo entre o nervo fibular, na região posterior da fíbula e a crista tibial (graus) e o ângulo entre o tubérculo de Gerdy e crista tibial (graus), região proximal da fíbula e a emergência do nervo fibular lateralmente (cm), ângulo entre o nervo e o eixo da fíbula (graus), distância entre a região proximal da fíbula e início da ramificação do nervo $(\mathrm{cm})$, distância entre o tubérculo de Gerdy e início da ramificação do nervo (cm), distância entre o tubérculo de Gerdy e ramo anterior recorrente $(\mathrm{cm})$ e número de ramos do nervo fibular (Figuras 1 e 2).

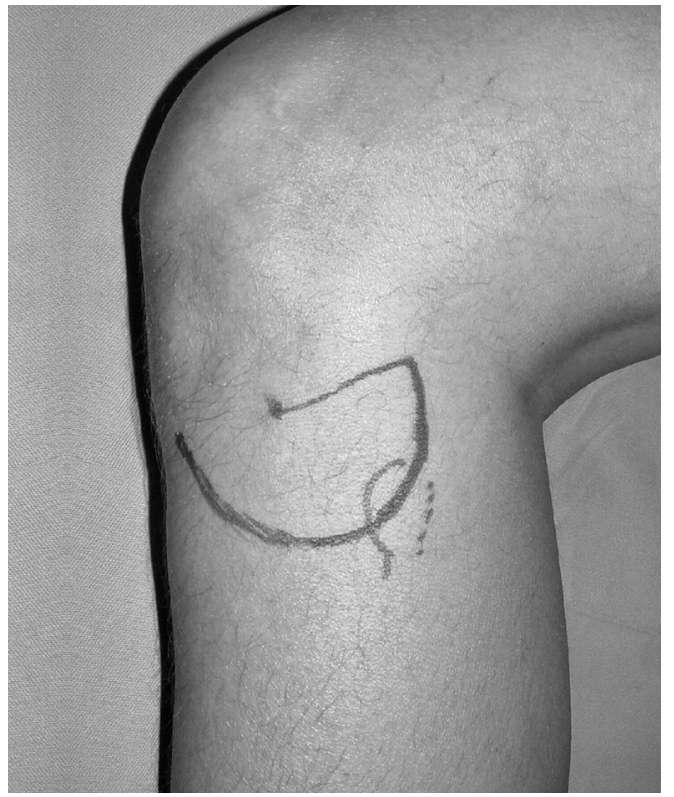

Figura 1 - Área demonstrando a zona de segurança de Gerdy

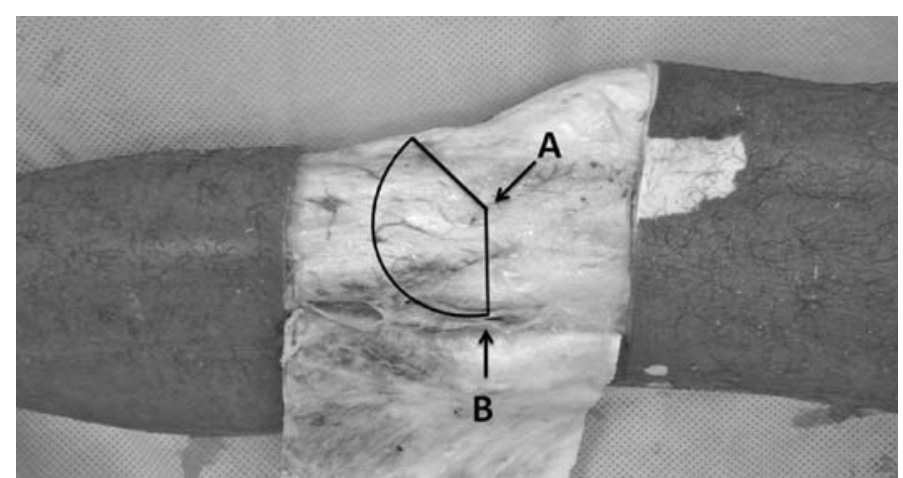

Figura 2 - Área demonstrando a zona de segurança de Gerdy no cadáver. A) Tubérculo de Gerdy. B) Nervo fibular comum

\section{Metodologia estatística}

A análise estatística foi realizada pelo teste dos postos sinalizados de Wilcoxon para avaliar a variação absoluta e relativa das medidas sobre o nervo fibular na face lateral do joelho antes e depois da dissecção. Foi aplicado teste não paramétrico, pois as medidas analisadas não apresentaram distribuição normal (distribuição gaussiana). O critério de determinação de significância adotado foi o nível de $5 \%$. A análise estatística foi processada pelo software SAS 6.04 (SAS Institute, Inc., Cary, NC).

\section{RESULTADOS}

Nosso estudo teve dois objetivos principais: analisar o posicionamento anatômico do nervo fibular e verificar a existência de uma variação entre as medidas antes e depois da dissecção de 50 espécimes anatômicos, tendo como limite o tubérculo de Gerdy. 
Objetivo 1: Perfil geral da casuística

Este estudo tem por finalidade traçar um perfil geral dos 50 espécimes anatômicos. A Tabela 1 fornece a média, desvio padrão (DP), mediana, mínimo e máximo das variáveis analisadas. Dos 50 espécimes anatômicos, 45 (90\%) são do sexo masculino e 26 (52\%) são do lado esquerdo.

Tabela 1 - Descritiva geral das variáveis analisadas

\begin{tabular}{l|c|c|c|c|c|c}
\hline \multicolumn{1}{c|}{ Variável } & $\mathbf{n}$ & Média & DP & Mediana & Mínimo & Máximo \\
\hline $\begin{array}{l}\text { Dist. entre reg. proximal - } \\
\text { emerg. nervo (cm) }\end{array}$ & 50 & 2,43 & 0,43 & 2,5 & 1,5 & 3,5 \\
\hline $\begin{array}{l}\text { Ângulo entre nervo - eixo } \\
\text { (graus) }\end{array}$ & 50 & 23,90 & 8,35 & 20 & 14 & 52 \\
\hline $\begin{array}{l}\text { Dist. entre reg. proximal - } \\
\text { início da ramificação (cm) }\end{array}$ & 50 & 2,23 & 0,63 & 2,2 & 1,2 & 5 \\
\hline $\begin{array}{l}\text { Distância entre TG - início da } \\
\text { ramificação (cm) }\end{array}$ & 50 & 4,71 & 0,50 & 4,6 & 3,8 & 5,5 \\
\hline $\begin{array}{l}\text { Distância entre TG - ramo } \\
\text { anterior (cm) }\end{array}$ & 50 & 4,45 & 0,52 & 4,4 & 3,5 & 5,5 \\
\hline \begin{tabular}{l} 
Número de ramos do fibular \\
\hline
\end{tabular} & 50 & 3,14 & 0,70 & 3 & 2 & 5 \\
\hline
\end{tabular}

DP: desvio padrão; TG: tubérculo de Gerdy

Fonte: Faculdade de Medicina de Petrópolis

Objetivo 2: Verificar se existe variação significativa nas medidas antes e depois da dissecção

As Tabelas 2, 3 e 4 fornecem a média, desvio padrão/ erro padrão (DP/EP), mediana, mínimo e máximo das medidas 1,2 e 3, respectivamente, antes e depois da dissecção, o correspondente delta absoluto e delta relativo (\%), e seu respectivo nível descritivo $(p)$ do teste dos postos sinalizados de Wilcoxon.

A medida 1 é a distância entre o tubérculo de Gerdy e a região posterior da fíbula $(\mathrm{cm})$; a medida 2 é o ângulo entre a região posterior da fíbula e a crista tibial (graus); e a medida 3 é ângulo entre o tubérculo de Gerdy e a crista tibial (graus).

O delta absoluto foi calculado pela seguinte fórmula:

Delta absoluto $=($ depois da dissecção - antes da dissecção).

O delta relativo (\%) foi calculado pela seguinte fórmula:

Delta relativo $(\mathbf{\%})=($ depois da dissecção - antes da dissecção) / antes da dissecção x 100.
Tabela 2 - Análise da variação da medida 1 antes e depois da dissecção

\begin{tabular}{c|c|c|c|c|c|c|c}
\hline Variável & $\mathbf{n}$ & Média & DP/EP & Mediana & Mínimo & Máximo & $\boldsymbol{p}$ valor \\
\hline Medida 1 antes (cm) & 50 & 4,17 & 0,58 & 4,2 & 2 & 5,2 & \\
\hline Medida 1 depois (cm) & 50 & 4,43 & 0,42 & 4,45 & 3,7 & 5,5 & \\
\hline Delta absoluto (cm) & 50 & 0,26 & 0,09 & 0,20 & $-1,1$ & 2 & $\mathbf{0 , 0 1 8}$ \\
\hline Delta relativo (\%) & 50 & 8,64 & 2,95 & 4,76 & $-22,4$ & 100 & $\mathbf{0 , 0 0 7}$ \\
\hline
\end{tabular}

DP: desvio padrão; EP: erro padrão apenas para os deltas

Fonte: Faculdade de Medicina de Petrópolis

Observou-se nesta amostra que existe aumento significativo na distância entre o tubérculo de Gerdy e a região posterior da fíbula (cm), após dissecção, em média, de $0,26 \mathrm{~cm}(p=0,018)$, o que corresponde a 8,6\% $(p=0,007)$, conforme ilustra a Figura 3.

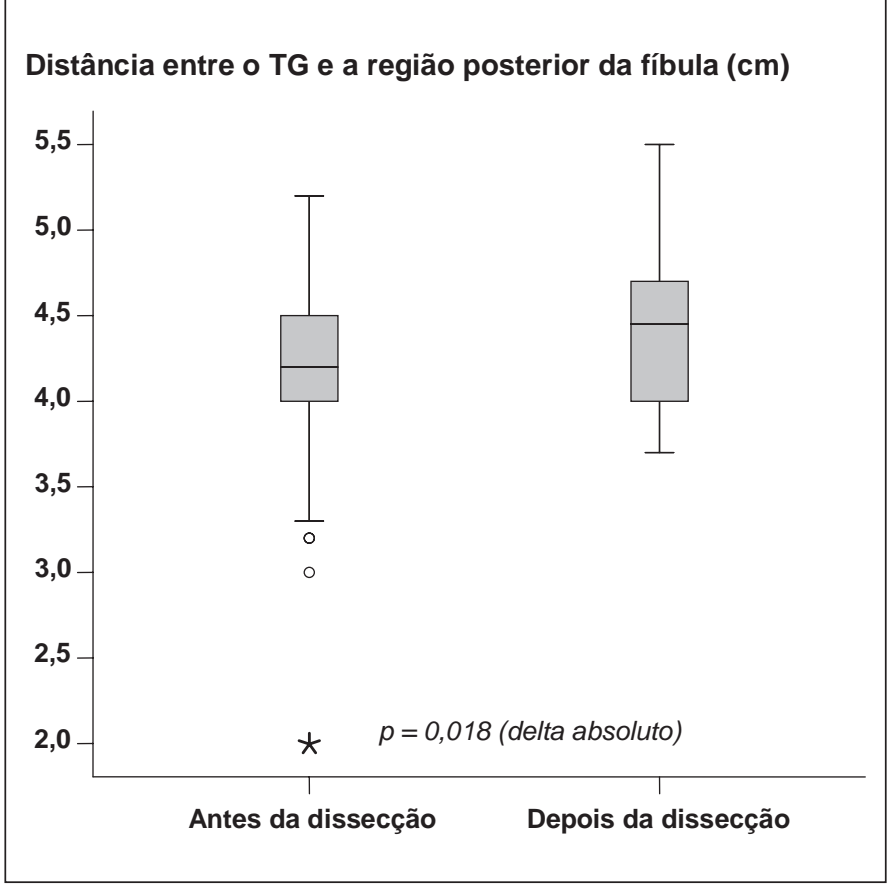

Figura 3 - Medida 1 antes e depois da dissecção

Tabela 3 - Análise da variação da medida 2 antes e depois da dissecção

\begin{tabular}{c|c|c|c|c|c|c|c}
\hline Variável & $\mathbf{n}$ & Média & DP/EP & Mediana & Mínimo & Máximo & $\boldsymbol{p}$ valor \\
\hline Medida 2 antes (graus) & 50 & 121,2 & 12,4 & 120 & 105 & 175 & \\
\hline Medida 2 depois (graus) & 50 & 118,3 & 14,0 & 112 & 100 & 170 & \\
\hline Delta absoluto (graus) & 50 & $-2,98$ & 1,79 & $-2,0$ & $-28,0$ & 33,0 & 0,047 \\
\hline Delta relativo (\%) & 50 & $-2,11$ & 1,45 & $-1,8$ & $-21,9$ & 29,5 & 0,060 \\
\hline
\end{tabular}

DP: desvio padrão; EP: erro padrão apenas para os deltas

Fonte: Faculdade de Medicina de Petrópolis 
Observou-se nesta amostra que existe diminuição significativa do ângulo entre a região posterior da fíbula e a crista tibial após dissecção, em média, de $3^{\circ}(p=$ $0,047)$, que corresponde a $2,1 \%(p=0,06)$, conforme ilustra a Figura 4.

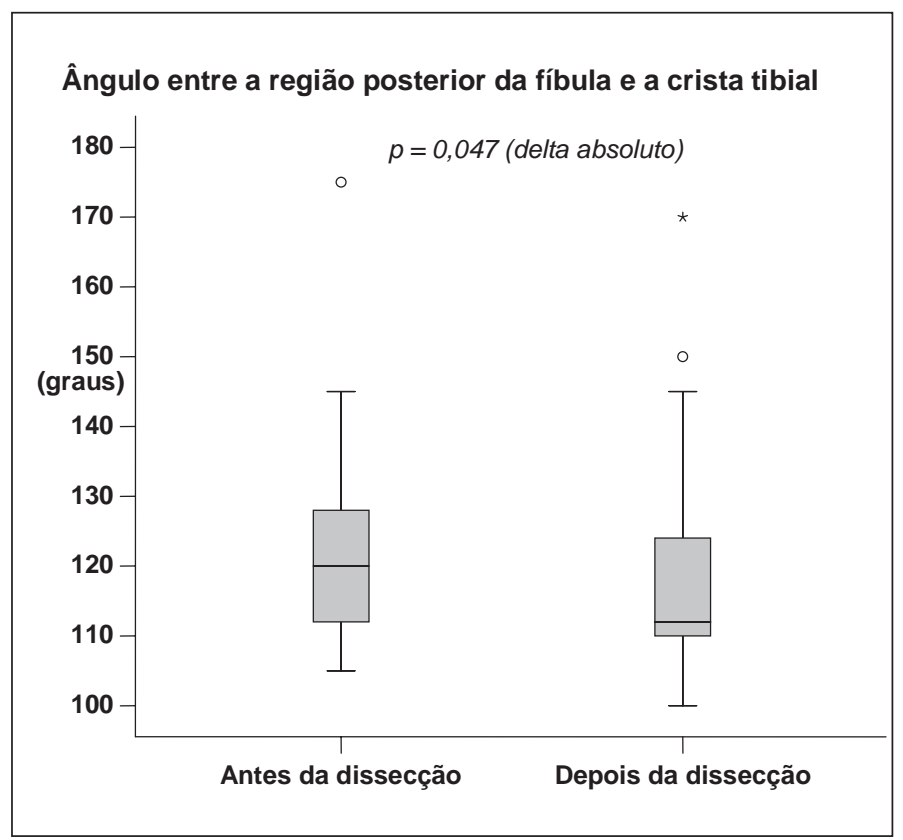

Figura 4 - Medida 2 antes e depois da dissecção

Tabela 4 - Análise da variação da medida 3 antes e depois da dissecção

\begin{tabular}{c|c|c|c|c|c|c|c}
\hline Variável & $\mathbf{n}$ & Média & DP/EP & Mediana & Mínimo & Máximo & p valor \\
\hline Medida 3 antes (graus) & 50 & 31,3 & 8,0 & 30 & 18 & 54 & \\
\hline Medida 3 depois (graus) & 50 & 29,8 & 7,5 & 28 & 20 & 48 & \\
\hline Delta absoluto (graus) & 50 & $-1,52$ & 1,19 & $-2,0$ & $-26,0$ & 20 & 0,14 \\
\hline Delta relativo (\%) & 50 & $-0,98$ & 4,07 & $-6,3$ & $-54,2$ & 80 & 0,29 \\
\hline
\end{tabular}

DP: desvio padrão; EP: erro padrão apenas para os deltas

Fonte: Faculdade de Medicina de Petrópolis

Observou-se, nesta amostra, que não existe variação significativa do ângulo entre o tubérculo de Gerdy e a crista tibial após dissecção. Houve diminuição de $1,5^{\circ}$ ( $p=0,14)$, que corresponde a $0,98 \%(p=0,29)$, em média neste estudo.

\section{DISCUSSÃO}

Várias são as doenças que se apresentam no terço proximal da tíbia e necessitam da interferência do cirurgião ortopédico. Dentre elas, estão a osteotomia proximal da tíbia como tratamento da deformidade em varo, secun- dária a osteoartrite do joelho ${ }^{(6,8)}$, tratamento das fraturas com fixadores externos ${ }^{(4,9-13)}$, fasciotomias após síndrome compartimental da perna ${ }^{(14-16)}$, biópsias percutâneas ao nível da região proximal da fíbula ${ }^{(5)}$ e osteotomia da fíbula $^{(2)}$. Portanto, o conhecimento da anatomia é muito importante, pois dano sobre o nervo fibular poderia trazer graves consequências funcionais ${ }^{(4,17,18)}$. Entretanto, a maioria das publicações do estudo anatômico tem descrito a localização do nervo fibular somente de forma linear, em duas dimensões, e em relação à cabeça da fíbula, o que faz com que o planejamento pré-operatório, em relação ao nervo, seja muito difícil ${ }^{(19)}$.

Kirgis e Albrecht ${ }^{(6)}$ desenvolveram uma escala longitudinal (mm), a partir do tronco profundo do nervo fibular, que ilustra as regiões de alto e baixo risco para lesão intraoperatória do nervo fibular.

Stitgen et al $^{(4)}$ descreveram a anatomia dos ramos do nervo fibular comum como "zona de segurança” para a colocação de fios percutâneos ao nível da região proximal da tíbia. Sua zona de segurança é localizada na região anterior à fíbula e a $2 \mathrm{~cm}$ de sua extremidade, criando uma área estimada de $4 \mathrm{~cm}^{(4,7)}$. Takeda et al ${ }^{(5)}$ definiram a zona de segurança para realizar biópsias no nível da região proximal da fíbula. Em seu estudo, o nervo foi visto cruzar a diáfise proximal da fíbula formando um ângulo de $23^{\circ}$.

Moskovich $^{(1)}$ realizou dissecções anatômicas na tentativa de mapear a trajetória do nervo fibular. Para obter essas medidas, Moskovich marcou o nervo com suturas de chumbo e fez radiografias. Em uma ilustração do seu trabalho, mostrou como o nervo e seu ramo anterior recorrente descrevem um círculo ao redor do tubérculo de Gerdy.

Segundo Rubel et al ${ }^{(19)}$, a zona de segurança ao nível da região proximal da tíbia, utilizando o tubérculo de Gerdy como limite, pode ser facilmente identificada e marcada pré-operatoriamente. Porém, temos que levar em consideração os pacientes com fraturas do terço proximal da tíbia, com grande edema, pacientes com deformidades prévias ou em pacientes obesos, onde a identificação do tubérculo de Gerdy se torna mais difícil.

A distância entre o tubérculo de Gerdy e a cabeça da fíbula determina um raio dessa zona de segurança. $\mathrm{O}$ trajeto do nervo fibular se mantém constante, formando um raio de aproximadamente $100^{\circ}$, começando na cabeça da fíbula e terminando na crista da tíbia. Nossos resultados demonstraram que, quando utilizamos o tubérculo de Gerdy como limite, existiu uma diferença significativa nas peças anatômicas, antes e depois da 
dissecção (121,2 ${ }^{\circ}$ antes de dissecar e $118,3^{\circ}$ depois). Após a dissecção, encontramos aumento, em média, de $0,26 \mathrm{~cm}(p=0,018)$, o que corresponde a $8,6 \%$ ( $p=$ 0,007 ) do raio entre o tubérculo de Gerdy e a região posterior proximal da fíbula, e diminuição do ângulo entre a região posterior da fíbula e a crista tibial, em média, de $3^{\circ}(p=0,047)$, que corresponde a $2,1 \%(p=0,06)$. Em nossas investigações e em outros estudos ${ }^{(4,19-21)}$ não encontramos ramos do nervo fibular dentro dessa área.

\section{CONCLUSÃO}

Apesar da diferença do raio entre o tubérculo de Gerdy e a região posterior proximal da fíbula e do ângulo entre a região posterior da fíbula e a crista tibial, antes e depois da dissecção, a zona de segurança de Gerdy pode ser considerada segura para os procedimentos ortopédicos na região proximal da tíbia, evitando danos ao nervo fibular e seus ramos.

\section{REFERÊNCIAS}

1. Moskovich R. Proximal tibial transfixion for skeletal traction. An anatomic study of neurovascular structures. Clin Orthop Relat Res. 1987;(214):264-8.

2. Rupp RE, Podeszwa D, Ebraheim NA. Danger zones associated with fibular osteotomy. J Orthop Trauma. 1994;8(1):54-8.

3. Soejima O, Ogata K, Ishinishi T, Fukahori Y, Miyauchi R. Anatomic considerations of the peroneal nerve for division of the fibula during high tibial osteotomy. Orthop Rev. 1994;23(3):244-7.

4. Stitgen SH, Cairns ER, Ebraheim NA, Niemann JM, Jackson WT. Anatomic considerations of pin placement in the proximal tibia and its relationship to the peroneal nerve. Clin Orthop Relat Res. 1992;(278):134-7.

5. Takeda A, Tsuchiya H, Mori Y, Tanaka S, Kikuchi S, Tomita K. Anatomical aspects of biopsy of the proximal fibula. Int Orthop. 2001;24(6):335-7.

6 . Kirgis A, Albrecht S. Palsy of the deep peroneal nerve after proximal tibial osteotomy. An anatomical study. J Bone Joint Surg Am. 1992;74(8):1180-5.

7. Slawski DP, Schoenecker PL, Rich MM. Peroneal nerve injury as a complication of pediatric tibial osteotomies: a review of 255 osteotomies. J Pediatr Orthop. 1994;14(2):166-72.

8. Aydogdu S, Yercan H, Saylam C, Sur H. Peroneal nerve dysfunction after high tibial osteotomy. An anatomical cadaver study. Acta Orthop Belg 1996;62(3):156-60.

9. El-Shazly M, Saleh M. Displacement of the common peroneal nerve associated with upper tibial fracture: implications for fine wire fixation. J Orthop Trauma. 2002;16(3):204-7.

10. Dendrinos GK, Kontos S, Katsenis D, Dalas A. Treatment of high energy tibial plateau fractures by the llizarov circular fixator. J Bone Joint Surg Br. 1996;78(5):710-7.

11. Gaudinez RF, Mallik AR, Szporn M. Hybrid external fixation of comminuted tibial plateau fractures. Clin Orthop Relat Res. 1996;(328):203-10.
12. Watson JT, Coufal C. Treatment of complex lateral plateau fractures using Ilizarov techniques. Clin Orthop Relat Res. 1998;(353):97-106.

13. Weiner LS, Kelley M, Yang E, Steuer J, Watnick N, Evans M, et al. The use of combination internal fixation and hybrid external fixation in severe proximal tibia fractures. J Orthop Trauma 1995;9(3):244-50.

14. Maquet $P$. The treatment of choice in osteoarthritis of the knee. Clin Orthop Relat Res. 1985;(192):108-12.

15. Garfin S. Mubarak SJ, Owen CA. Exertional anterolateral-compartment syndrome. Case report with fascial defect, muscle herniation, and superficial peroneal-nerve entrapment. J Bone Joint Surg Am. 1977;59(3):404-5.

16. Mubarak SJ, Owen CA. Double-incision fasciotomy of the leg for decompression in compartment syndromes. J. Bone Joint Surg Am. 1977;59(2):184-7.

17. Makarov MR, Delgado MR, Birch JG, Samchukov ML. Intra-operative SSEP monitoring during external fixation procedures in the lower extremities. J Pediatr Orthop. 1996;16(2):155-60.

18. Loredo R, Hodler J, Pedowitz R, Yeh LR, Trudell D, Resnic D. MRI of the common peroneal nerve: normal anatomy and evaluation of masses associated with nerve entrapment. J Comput Assist Tomogr. 1998;22(6):925-31.

19. Rubel IF, Schwarzbard I, Leonard A, Cece D. Anatomic location of the peroneal nerve at the level of the proximal aspect of the tibia: Gerdy's safe zone. J Bone Joint Surg Am. 2004;86(8):1625-8.

20. Adkison DP, Bosse MJ, Gaccione DR, Gabriel KR. Anatomical variations in the course of the superficial peroneal nerve. J Bone Joint Surg Am. 1991;73(1):112-4.

21. Dellon AL, Ebmer J, Swier P. Anatomic variations related to decompression of the common peroneal nerve at the fibular head. Ann Plast Surg. 2002;48(1):30-4. 